\title{
Roles of Dynamic State Estimation in Power System Modeling, Monitoring and Operation
}

\author{
IEEE Task Force on Power System Dynamic State and Parameter Estimation \\ Junbo Zhao (TF Chair), Senior Member, IEEE, Marcos Netto, Member, IEEE, Zhenyu Huang, Fellow, IEEE, \\ Samson Shenglong Yu, Member, IEEE, Antonio Gómez-Expósito, Fellow, IEEE, Shaobu Wang, Senior \\ Member, IEEE, Innocent Kamwa, Fellow, IEEE, Shahrokh Akhlaghi, Member, IEEE, Lamine Mili, Life \\ Fellow, IEEE, Vladimir Terzija, Fellow, IEEE, A. P. Sakis Meliopoulos, Fellow, IEEE, Bikash Pal, Fellow, IEEE, \\ Abhinav Kumar Singh, Member, IEEE, Ali Abur, Fellow, IEEE, Tianshu Bi, Senior Member, IEEE, Alireza \\ Rouhani, Member, IEEE
}

\begin{abstract}
Power system dynamic state estimation (DSE) remains an active research area. This is driven by the absence of accurate models, the increasing availability of fast-sampled, timesynchronized measurements, and the advances in the capability, scalability, and affordability of computing and communications. This paper discusses the advantages of DSE as compared to static state estimation, and the implementation differences between the two, including the measurement configuration, modeling framework and support software features. The important roles of DSE are discussed from modeling, monitoring and operation aspects for today's synchronous machine dominated systems and the future power electronics-interfaced generation systems. Several examples are presented to demonstrate the benefits of DSE on enhancing the operational robustness and resilience of 21st century power system through time critical applications. Future research directions are identified and discussed, paving
\end{abstract}

This work was partially supported by US Department of Energy under Advanced Grid Modernization Program.

Junbo Zhao is with Department of Electrical and Computer Engineering, Mississippi State University, Starkville, MS, 39759 (e-mail: junbo@ece.msstate.edu).

Marcos Netto is with National Renewable Energy Laboratory, Golden, CO 80401 USA (e-mail: Marcos.Netto@nrel.gov).

Zhenyu Huang and Shaobu Wang are with the Pacific Northwest National Laboratory, Richland, WA 99352, USA (email: zhenyu.huang@pnnl.gov, Shaobu.Wang@pnnl.gov).

Samson Shenglong Yu is with School of Engineering, Deakin University, Melbourne, Victoria, Australia (e-mail: samson.yu@ deakin.edu.au).

Antonio Gómez-Expósito is with the Department of Electrical Engineering, University of Seville, Seville 41092, Spain (e-mail: age@us.es).

Innocent Kamwa is with Hydro-Quebec/IREQ, Power Systems and Mathematics, Varennes, Canada (e-mail: kamwa.innocent@ireq.ca).

Shahrokh Akhlaghi is with Southwest Power Pool, Little Rock, AR 72223, USA (e-mail: sakhlaghi@spp.org).

Lamine Mili is with the Bradley Department of Electrical and Computer Engineering, Virginia Polytechnic Institute and State University, Falls Church, VA 22043, USA (e-mail: 1mili@vt.edu).

Vladimir Terzija is with the School of Electrical and Electronic Engineering, The University of Manchester (email: vladimir.terzija@manchester.ac.uk).

A. P. S. Meliopoulos is with the School of Electrical and Computer Engineering, Georgia Institute of Technology, Atlanta, GA 30339 USA (email: sakis.m@gatech.edu).

Bikash Pal is with the Department of Electrical and Electronic Engineering, Imperial College London SW7 2BT, U.K. (e-mail: b.pal@imperial.ac.uk).

Abhinav Kumar Singh is with the School of Electrical and Electronic Engineering, University of Southampton, UK (e-mail: aks@ecs.soton.ac.uk).

Ali Abur is with the Department of Electrical and Computer Engineering, Northeastern University, Boston, MA, USA (e-mail: abur@ece.neu.edu).

T. Bi is with the State Key Laboratory of Alternate Electrical Power System with Renewable Energy Sources, North China Electric Power University, Beijing 102206, China (e-mail: tsbi@ncepu.edu.cn).

Alireza Rouhani is with Dominion Energy, Richmond, VA 23219, USA (e-mail: Alireza.Rouhani@dominionenergy.com). the way for developing the next generation of energy management systems and novel system monitoring, control and protection tools to achieve better reliability and resiliency.

Index Terms-Dynamic state estimation, Kalman filtering, low inertia, monitoring, parameter estimation, power system stability, synchronous machines, synchrophasor measurements, converter interfaced generation, static state estimation.

\begin{tabular}{ll} 
COI & \multicolumn{1}{c}{ ACRONYMS } \\
DAE & Center of inertia \\
DERs & Differential-algebraic equations \\
DFIG & Doubly-fed induction generator \\
DFR & Digital fault recorder \\
DSA & Dynamic security assessment \\
DSE & Dynamic state estimation \\
EKF & Extended Kalman filter \\
EMS & Energy management system \\
EnKF & Ensemble Kalman filter \\
LSE & Linear state estimation \\
MU & Merging unit \\
NERC & North American Electric Reliability Corp. \\
PF & Particle filter \\
PMU & Phasor measurement unit \\
RESs & Renewable energy sources \\
PSS & Power system stabilizer \\
ROCOF & Rate of change of frequency \\
SCADA & Supervisory control and data acquisition \\
SSE & Static state estimation \\
UKF & Unscented Kalman filter
\end{tabular}

\section{INTRODUCTION}

D YNAMIC state estimation (DSE) [1] is going to be very useful for time critical monitoring, control, and protection of future electric power grids. This is largely due to the changes in generation mixes and load compositions, particularly the increasing penetration of intermittent, stochastic and power electronics-interfaced non-synchronous renewable generation and distributed energy resources (DERs) [2]. In this context, where the system operating point changes more often and more rapidly, tracking the system dynamic state variables is of critical importance. Recall that dynamic state 
variables are the ones associated with the time derivatives in the set of differential-algebraic equations describing power system dynamics [3]. The dynamic state variables of the synchronous generators correspond to their electromagnetic and electromechanical processes, as well as their controllers [4]. As for the non-synchronous generations, the dynamic state variables are associated with the primary source of energy, e.g. solar photovoltaic arrays, batteries and wind turbines, as well as their controllers. Examples include the frequency at the point of common coupling between the power converter and the electric grid, the electric current in the converter, and the pitch angle of wind turbines. Furthermore, there exists an important challenge in the development and maintenance of accurate models for power electronics-interfaced devices. DSE can be developed to validate the models and to estimate unknown or incorrect parameters.

Despite being initially mentioned in the 1970s [5], it was only in recent years that the power system community has picked up the momentum in DSE research. Part of the reason was the lack of appropriate metering infrastructure, like phasor measurement units (PMUs) and merging units (MUs) that are being widely deployed to capture the appropriate dynamics in power systems [6], [7]. Feasibility studies using PMU measurements for DSE are reported in [8], and subsequently, various Kalman filtering techniques, such as Extended Kalman Filter (EKF), Unscented Kalman Filter (UKF) [9], Ensemble Kalman Filter (EnKF), Particle Filter (PF) and their variants [10] have been applied to DSE. Data-driven DSE [11], observability analysis to guide measurement selection [12], and the enhancement of robustness against bad data and parameter errors are also developed in [13]-[15]. Readers may refer to [4] for a comprehensive summary of DSE algorithms.

Several online tasks can benefit from DSE but have not adequately deliberated the need for it. An example is the dynamic security assessment (DSA). Today's DSA tool assumes a steady-state initial condition [16], which would yield inaccurate results in the presence of fluctuations from DERs and loads; conversely, DSE could directly estimate the dynamic states and therefore provide more accurate initialization conditions for DSA [17]. Furthermore, for synchronous generators, numerous developed control schemes rely on power system stabilizers (PSS) to damp out oscillations. These control schemes use the rotor's speed as input. The direct use of measurements obtained from a meter installed on the shaft of the machine is not reliable; instead, the current practice is to use the compensated frequency [18], which is calculated using the voltage and current measured at the generators' terminal. However, the calculation of the compensated frequency is significantly affected under transient conditions [19], which leads to sub-optimal control response of the PSS. If DSE is deployed for frequency estimation, this issue can be effectively resolved. The rate of change of frequency (ROCOF) estimation can also be formulated in the state- space model for DSE [20]. Note that, in these examples, traditional static state estimation (SSE) could not provide reliable system dynamic states and quantities. Therefore, it is of critical importance to understand the role of DSE in power systems from various aspects. While SSE has been in energy management systems (EMS) for decades and its roles and implementation requirements are well established, the same does not apply for DSE. There is a large gap in understanding different roles of SSE and DSE and how DSE can be implemented in practice.

This paper summarizes the joint efforts of the Task Force in addressing the aforementioned gaps and yields the following contributions:

- A comprehensive clarification of the roles of DSE from the power system modeling, monitoring, and operation perspectives are provided;

- Comparisons between SSE and DSE in practical implementations are thoroughly discussed, including measurement configurations, model requirements, software support and potential applications based on the estimation results;

- Illustrative examples and summary frameworks have been presented to demonstrate the roles of DSE.

- The future applications are discussed to shed light on the transition from today's EMS to its next generation.

The remainder of this paper is organized as follows. Section II compares DSE and SSE from the practical implementation requirement and application aspects. Section III summarizes the role of DSE in different applications. Conclusions and directions for future research are given in Section IV.

\section{Comparative Overview of SSE AND DSE}

SSE has become a widely used tool in today's EMS, while DSE is a new tool for the industry and system operators. It is essential to clarify their implementation and functionality differences and, at the same time, enable a clear path transition from SSE-based EMS to the future DSE-based EMS with power electronics-dominated power systems.

\section{A. Implementation Differences}

SSE and DSE have different requirements with respect to measurements, models, observability, execution rate, outputs and applications. These differences are summarized in Table I. SSE mostly relies on SCADA measurements that are updated every 2-5s and some PMU measurements to gain more redundancy. But since SCADA measurements are not timesynchronized while those of PMUs are, effectively integrating those two sources of data needs to be taken care of. By contrast, for DSE, time-synchronized measurements with a reporting rate of 30 to 240 samples per second are used and they might come from PMUs and digital fault recorders (DFRs). Furthermore, there is a significant difference in the observability theory for SSE and DSE [12], [21]. For SSE, the topological- or numerical-based observability analysis typically determines whether the system is observable or not. If the system is unobservable, observable islands may be determined [22]. Furthermore, in the presence of ampere measurements, where multiple solutions may exist depending on the nature of the remaining measurements and the loading point, the observability analysis can also inform whether there are multiple solutions or not to the SSE problem. A network can be uniquely observable for heavily loaded cases, but "unobservable" (i.e., undefined Jacobian) when the ampere measurement value is nearly zero (unloaded line), which leads to a $0 / 0$ indeterminate 
form. Thus, the answer depends on the line loading but is typically binary. By contrast, in DSE, one may refer to strongly or weakly or not observable systems. One way to quantify this is to compute the smallest singular value of the observability matrix from the Lie derivatives [12]. Higher (lower) values of the smallest singular value of the observability matrix indicates stronger (weaker) observability for a given measurement set. Furthermore, due to the nonlinear and time-dependent nature of the problem, observability results are time-varying. It is worth noting that under certain conditions, the system might be unobservable, but still detectable [23] for DSE. Detectability is a slightly weaker notion than observability. A system is detectable if all the unobservable states are stable. To enhance the observability of power system dynamic state estimation, some optimal PMU placement strategies [21], [24] have been developed. This work can be further investigated considering the robustness against data quality issues, model uncertainties, etc. Also, each prediction-correction step of the DSE must be numerically solved faster than the PMUs/DFRs/MUs scan rate, thereby posing some challenges on the computational power. To address this issue, decentralized/distributed DSE or parallel computing technique for centralized DSE is usually suggested [4]. Another important issue that merits attention is the algorithm convergence. It has been shown for SSE that under heavy loading and topology error conditions, there may be convergence problems [25], [26]. Therefore, both numerical and statistical robustness need to be enhanced to deal with that. The data quality issues, inappropriate initialization and model errors/uncertainties may lead to convergence issues for DSE. Although several theoretical works have been carried out to prove the convergence of various types of nonlinear Kalman filters [27]-[29] under some assumptions, their applications to power systems under various conditions need thorough investigations. This is because some assumptions in the theoretical developments may not always hold. Thus, nonlinear Kalman filters without linearization are recommended for practical implementations, such as UKF, EnKF, PF, etc.

The outcome of SSE and DSE are also different. SSE provides estimates of the bus voltage magnitudes and phase angles, which are the algebraic variables; on the other hand, DSE provides estimates of the dynamic state variables, such as those associated with generators/dynamic loads/DERs. There is also joint DSE that estimates dynamic and algebraic variables simultaneously [4]. For some PMU observable networks, linear state estimation (LSE) that keeps up with the PMU refreshing rate is developed [30]. However, it does not track the actual system dynamics.

When implementing SSE and DSE, the models used are different. In SSE, the generators and loads are simply modeled

\footnotetext{
${ }^{1}$ Topological observability analysis provides a binary answer as the first byproduct, but it gives additional valuable information, such as which buses (islands) are observable or which pseudo-measurements should minimally be added to restore full observability. Numerical observability analysis, being based on the factorization of the Jacobian or Gain matrices, provides a "spectrum" or range of observability answers, depending on the condition number of the matrix being factorized. A network can be topologically observable but not algebraically (numerically) observable, owing to abnormal network parameters or measurement weights. In some cases, a network can be numerically observable for a certain combination of weights, but not for others. This is not exactly "binary".
}

TABLE I

COMPARISON BETWEEN SSE AND DSE.

\begin{tabular}{|c|c|c|}
\hline & SSE & DSE \\
\hline Measurements & $\begin{array}{l}\text { From the SCADA } \\
\text { (every } \sim 2-10 \mathrm{sec} \text {.) }\end{array}$ & $\begin{array}{l}\text { From PMU/DFR/MU } \\
\text { (every } 1 / 30 \sim 1 / 240 \mathrm{sec} \text {.) }\end{array}$ \\
\hline Observability & $\begin{array}{c}\text { Binary }{ }^{1} \\
\text { (observable or not) }\end{array}$ & $\begin{array}{c}\text { Time-varying } \\
\text { (Strong/weak/not observable) }\end{array}$ \\
\hline Update speed & $\begin{array}{c}1 \text { snapshot } \\
\text { (every } \sim 2-10 \mathrm{sec} . \text { ) }\end{array}$ & $\begin{array}{l}1 \text { prediction }+1 \text { filtering } \\
\text { step of the Kalman filter } \\
\text { (every }<1 / 30 \text { sec.) }\end{array}$ \\
\hline Models & $\begin{array}{l}\text { Algebraic power } \\
\text { flow equations }\end{array}$ & $\begin{array}{c}\text { Differential-algebraic } \\
\text { equations }\end{array}$ \\
\hline Framework & $\begin{array}{l}\text { Mostly centralized } \\
\text { or distributed }\end{array}$ & $\begin{array}{c}\text { Centralized \& } \\
\text { distributed/decentralized }\end{array}$ \\
\hline Outputs & $\begin{array}{l}\text { Algebraic variables } \\
\text { (voltage magnitudes } \\
\text { and angles) }\end{array}$ & $\begin{array}{c}\text { Dynamic variables } \\
\text { (machine/ dynamic load/ } \\
\text { DERs dynamic variables) }\end{array}$ \\
\hline Applications & $\begin{array}{l}\text { Monitoring and control } \\
\text { (operator in the loop) }\end{array}$ & $\begin{array}{l}\text { Monitoring (operator in } \\
\text { the loop), control and } \\
\text { adaptive protection } \\
\text { (operator out of the loop) }\end{array}$ \\
\hline
\end{tabular}

by power injections and, hence, the system model used for SSE is represented using algebraic equations. While for DSE, the generators/dynamic loads/DERs, etc., and their controllers are represented by a set of DAEs. Note that it is not required to have PMUs installed at each generator terminal; if the generator terminal is observable via a local LSE, the DSE can be implemented.

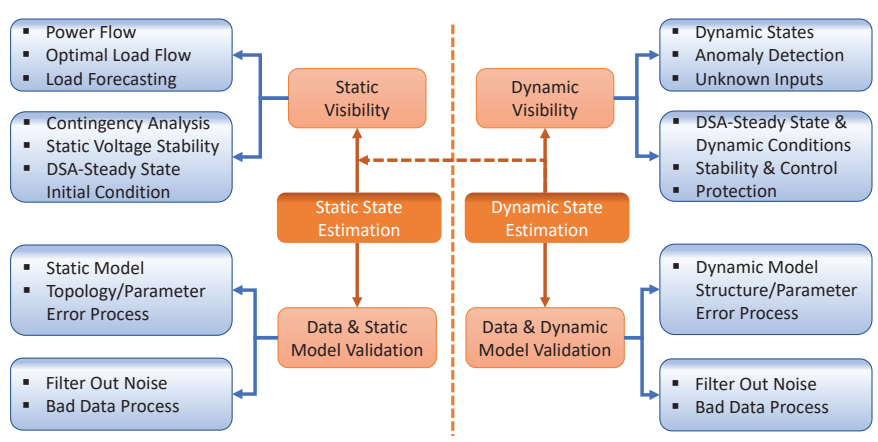

Fig. 1. Functional schematic comparisons between SSE and DSE.

\section{B. Functionality Differences}

From the functionality perspective, both SSE and DSE have two essential roles, namely improving the targeted system visibility and validating the used data and models, which are summarized in Fig. 1. Since SSE is the snapshot-based static view of the system algebraic variables, its outputs allow the calculations of power injections and power flows across the systems. This provides the database for many applications in today's EMS, including system operations, such as power flow, optimal power flow, load forecasting, etc., as well as security assessment, i.e., static contingency and static voltage stability analysis. By contrast, DSE relies on fast sampled and time synchronized measurements to track system dynamic changes, thus providing dynamic visibility, such as dynamic states, anomaly detection, unknown inputs estimation, etc. [31]-[33]. This dynamic information further allows other related applications, i.e., DSA [17], rotor angle 
stability assessment [33], adaptive power system protection [34], and so on. In terms of data and model validation for $\mathrm{SSE}$, it relies on the measurement residual-based statistical test or robust estimation criteria for bad data processing and measurement model parameter error identifications. The bad data are caused by gross measurement errors or data transfer errors while model parameter errors can be due to transmission line parameters and topology inaccuracies. Since DSE relies on a set of DAEs, besides those parameter errors previously mentioned, there are also model errors in equations that govern the system dynamics, e.g., generator models and their related controllers. These errors are classified into two categories: innovation outliers, which are caused by parameter and/or input errors in the set of DAEs; and structural outliers, which are caused by a model structure that does not reflect well the system dynamics [35]. Note that the DSE outputs can be used to calculate the system algebraic variables as well. Therefore, the SSE functions can benefit from DSE. And vice versa, the information provided by the SSE can be used to warm start the DSE process before an event occurs.

Remark: with the increasing deployment of PMUs, many high voltage transmission systems are already observable with only PMU measurements, such as that of the Dominion Energy, the $765 / 345 / 230 \mathrm{kV}$ power grid in New York, and the $345 \mathrm{kV}$ power grid in New England. As a result, the legacy nonlinear SSE algorithms can now be reformulated as the LSE [30]. As long as the computational power is sufficient, the LSE can be updated with the scan rate of PMUs but its results are also restricted to algebraic variables-based applications. Compared with the SCADA-based SSE, an LSE allows for faster real-time contingency analysis and voltage stability assessment, besides area angle limit monitoring. By comparing LSE with DSE, LSE does not touch the dynamic equations and can not provide the real-time picture of system dynamic states and the related controllers. For example, the rotor speed information is not available from LSE and thus many applications based on them cannot be done. The importance of dynamic states for power system monitoring, visibility and operation will be highlighted in Section III. In summary, the fundamental differences in terms of potential applications are that LSE only deals with algebraic variables while DSE provides both dynamic and algebraic variables of the system.

\section{Practical Implementation of DSE}

There are several vendors who offer commercial software for SSE implementation while there is no commercial software for DSE. However, we can leverage the capabilities of existing commercial tools and enable the DSE implementation. The practical implementation of DSE is shown in Fig. 2, where the system is modeled by the following state-space model after time discretization of DAEs, and considering the available measurements:

$$
\begin{aligned}
\boldsymbol{x}_{k} & =\boldsymbol{f}\left(\boldsymbol{x}_{k-1}, \boldsymbol{y}_{k-1}, \boldsymbol{u}_{k}, \boldsymbol{p}_{k}\right)+\boldsymbol{w}_{k}, \mathbb{E}\left[\boldsymbol{w}_{k} \boldsymbol{w}_{k}^{\top}\right]=\boldsymbol{Q}_{k} \\
\boldsymbol{z}_{k} & =\boldsymbol{h}\left(\boldsymbol{x}_{k}, \boldsymbol{y}_{k}, \boldsymbol{u}_{k}, \boldsymbol{p}_{k}\right)+\boldsymbol{v}_{k}, \mathbb{E}\left[\boldsymbol{v}_{k} \boldsymbol{v}_{k}^{\top}\right]=\boldsymbol{R}_{k}
\end{aligned}
$$

where $\boldsymbol{x}_{k}$ and $\boldsymbol{y}_{k}$ represent system dynamic and algebraic state vectors, respectively; $\boldsymbol{z}_{k}$ is the measurement vector from and/or DFRs and/or MUs; $\boldsymbol{u}_{k}$ is the system input vector that drives the state transition; $\boldsymbol{p}_{k}$ denotes the system parameters; $\boldsymbol{f}$ and $\boldsymbol{h}$ are vector-valued nonlinear functions; $\boldsymbol{w}_{k}$ and $\boldsymbol{v}_{k}$ are the system process and measurement error vector, respectively, with covariance matrices $\boldsymbol{Q}_{k}$ and $\boldsymbol{R}_{k}$.

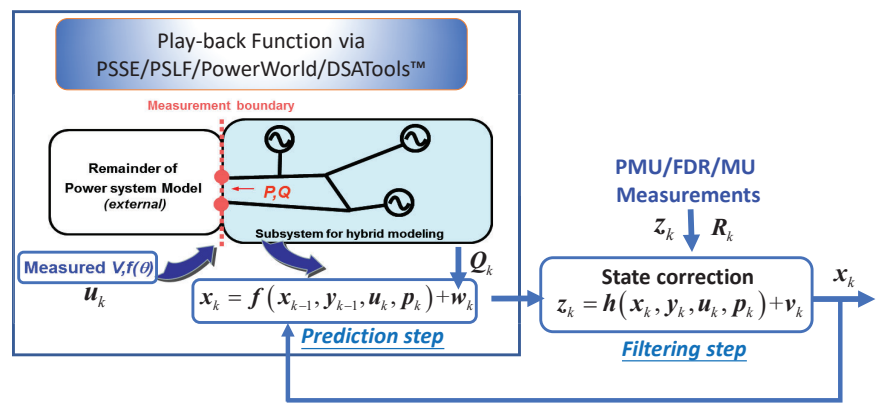

Fig. 2. Implementation of DSE using existing commercial tools.

It is clear from Fig. 2 that the implementation of DSE has two main steps, namely the state prediction via (1) to obtain predicted state at time $k$ and the state correction step that integrates the state prediction and measurements in (2) for filtering. It is interesting to note that the widely used commercial software, such as PSSE/ PSLF/ PowerWorld/ DSATools have the so-called event playback functions available. This play back function allows us to implement equation (1) automatically. The main idea of the play-back function is to take the measured voltage phasor as the input $\boldsymbol{u}_{k}$ of (1) and obtain the predicted state vector. Then, by resorting to the Kalman filter framework, the predicted state vector is integrated together with the received measurement vector in (2) for the estimation of dynamic states. To fully leverage this playback function, it is suggested to use nonlinear Kalman filter techniques that do not require linearization, such as UKF, EnKF, PF and their variants. Another useful implementation of DSE is to take unknown dynamic states or control inputs as additional state variables for joint estimation. This is because there may be a lack of accurate controller information or the control models may be of poor quality. Therefore, it can be concluded that only the filtering step needs to be coded along with the playback function for practical DSE implementation. In other words, no major changes are required and only additional module needs to be added to make the smooth transition of play-back function for practical DSE implementation. It should be noted that we do not need to wait for an event to trigger the DSE. As long as the measured vector $\boldsymbol{u}_{k}$, typically the voltage magnitudes and angles/frequency, are fed into the playback function, the DSE can be implemented to continuously monitor the dynamic states. For a successful implementation of DSE, the observability analysis using Lie derivatives should be carried out first and the required measurements should be provided [12]. Besides the basic observability requirement, higher measurement redundancy would lead to better estimation accuracy and capability of handling bad data. Some works [21], [24] about PMU placement strategy for improving the observability of dynamic state estimation have been investigated. 


\section{Roles of DSE in Power Systems Modeling, MONITORING AND OPERATION}

Three major application areas that can potentially benefit from DSE have been identified and elaborated in this paper, namely: a) modeling, b) monitoring via the enhanced dynamic visibility, and c) operation. Each of them is discussed below.

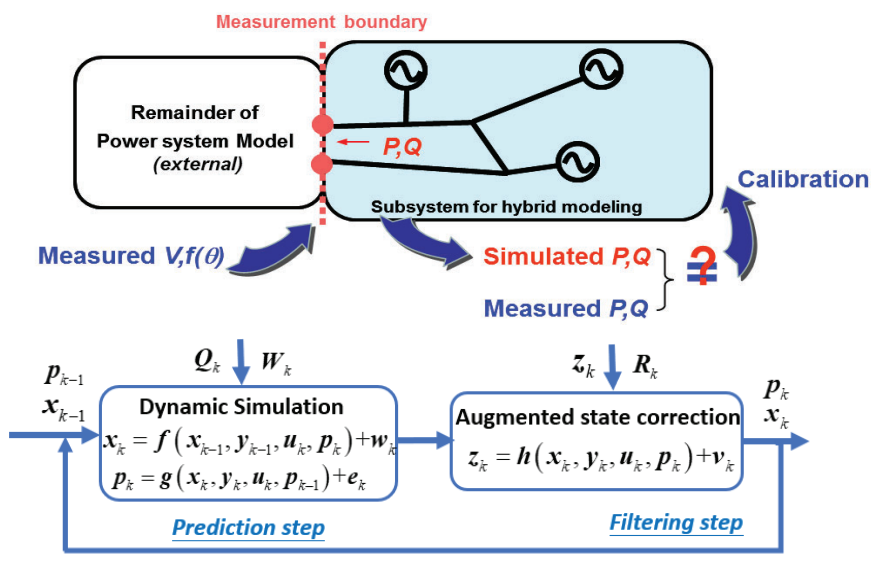

Fig. 3. DSE-enabled model validation and calibration.

\section{A. Modeling}

Following the 2003 Northeastern U.S. and Eastern Canada blackout, the North American Electric Reliability Corporation (NERC) has been developing standards for periodical model validation [36]. The model validations include synchronous generator and its related controllers, wind farms, solar farms, dynamic loads, etc. In this section, we first present the generator model validation and calibration framework, followed by other DSE-based applications from modeling perspective.

1) Generator Model Validation and Parameter Calibration: In the existing commercial software, the "event playback" function is leveraged to validate dynamic models using PMU data. The key idea is to take the measured generator terminal voltage magnitude/phase angle or frequency as model inputs to obtain model outputs of real power $P$ and reactive power $Q$. These responses are compared with the measured $P$ and $Q$ to validate the model accuracy [37]-[39]. When the model of the subsystem is accurate, the simulation responses should match statistically well the actual responses. By contrast, when significant mismatches are observed, the model is considered inadequate and parameter calibration is needed. This process can be found in the upper part of Fig. 3, where $e_{k}$ is the parameter error with covariance matrix $\boldsymbol{W}_{k}$ and $\boldsymbol{g}$ is the parameter transition function. The differences between Figs. 2 and 3 include: 1) statistical test between the simulated responses and the measured response to validate the model and 2) augmenting suspicious parameters obtained from sensitivity analysis and engineering judgement with the system dynamic states for joint estimation. Note that the criterion for model validation is a byproduct of DSE, namely the innovation vector-based statistical test.

To calibrate the system models, both the system model structure and parameters need to be carefully investigated. Traditionally, they can be found from manufacturer's databases.
However, parameters might drift during operations due to a variety of factors, e.g., environmental changes, aging process, and coupling effects. Taking generators offline for testing and parameter calibration is costly and affects the system reliability. Therefore, online calibration has been an important application of DSE. Since not all parameters are identified using the present measurement sets, it is, therefore, critical to select appropriate candidate parameters before starting a parameter calibration procedure. The calibration typically includes four main steps [37], [38], namely 1) initial checks, 2) sensitivity analysis, 3) parameter estimation and 4) parameter validation using other disturbances. The first step is to eliminate obvious errors in parameters that are not realistic, such as fractional values for the model flags that should be integers, parameter values outside the normal ranges, swapped values for limiters, and incorrect statuses of controllers. The sensitivity analysis provides a guideline in determining parameter sensitivity for different disturbances and identifying the candidate parameters for calibration. The selected parameter vector $\boldsymbol{p}$ is augmented with the original state vector for joint estimation as shown in the lower part of Fig. 3. The joint DSE methods are based on, e.g., EKF, UKF, constrained UKF and many other Kalman filter variants. After calibration, it is necessary to perform model validation using different events so that the identified parameters are not local optima. If the model deficiency for other events is detected, the calibration process should be repeated until there are no inconsistent responses between models and measurements.

Model validation process can be used to guide parameter estimation via DSE. There can be direct use of DSE to track parameter changes or identification of unknown machine parameters. For example, the UKF algorithms have been leveraged in [40], [41] to estimate the parameters of synchronous machine. The measurements may include voltages and current signals from the stator and the field winding or PMUs. A constrained UKF is further developed in [42] to narrow down the parameter search space and yield better parameter estimation results. DSE-based generator inertia, sub-transient reactance and governor dead-band estimation approaches can be found in [43], [44].

2) Other Parameter Estimation Applications: Besides synchronous machine model validation and calibration, the targeted dynamic components can be dynamic loads, wind farms, and other power electronics-interfaced DERs [45]-[48] as long as they are appropriately described by DAEs and their related local fast measurements are available. In [45], the first-order exponential dynamic load model is organized as the state-space model and an UKF is used to track the unknown parameters. Motivated by the parameter estimation of synchronous machines, the state-space model of DFIG is derived and the unknown parameters are augmented with its dynamic states for joint estimation via EnKF or UKF [47], [48]. It is worth noting that there are only few works on DSE for parameter estimation of dynamic loads and solar/wind farms. As the technologies of DSE for synchronous machines are becoming mature, extending them for dynamic components, especially the power electronics-based DERs and dynamic loads, is worth further investigations. The power system coherency identifi- 
cation would also benefit from the estimated dynamic states, especially the rotor angles and speeds [49], [50]. The identified coherency allows the development of model reduction for stability assessment and islanding control. Another potential application of DSE is the dynamic reduction of large power systems [51], where the equivalent model parameters can be estimated together with the machine dynamic states.

\section{B. Monitoring}

One of the key DSE applications is to provide enhanced dynamic visibility for power system monitoring. Part of the dynamic visibility of DSE has been shown in Fig. 1 but a more detailed summary includes seven key functions: i) dynamic state trajectory tracking, ii) oscillation monitoring, iii) bus frequency, ROCOF and center of inertia (COI) frequency estimation, iv) data quality detection and correction, including cyber attacks, v) unknown control inputs identification, vi) anomaly detection and vii) other applications that involve larger time constants than the electromechanical dynamics. The dynamic state trajectory tracking is a natural product of DSE as it provides the time series of the generator and its controller states in the presence of system disturbance. Among these, the generator rotor speed and angle have been widely used in power system for oscillation detection [52]. For example, in damping-torque-based forced oscillation source location, the rotor speed and angle are required [53]. The estimated machine rotor speeds also allow us to resort to the frequency divider for bus frequency estimation [54]. This interesting result reveals that if the system machine rotor speeds are available, all bus frequencies can be estimated. Since the number of machines is much smaller than the number of buses, it significantly reduces the requirement of PMU installations for bus frequency monitoring. The realtime COI frequency plays an important role in power system stability analysis and control. Via the DSE outputs, we can obtain the COI frequency at the same refreshing rate of the PMU measurements. This provides the online reference frequency for control [55]. Note that a widely used industry practice is to leverage generator terminal voltage and current measurements to calculate an approximated internal machine rotor speed. However, its accuracy is questionable as can be seen in the following example: a three-phase short-circuit occurs at Bus 16 of the IEEE 39-bus system on $t=0.5 \mathrm{~s}$ and the fault is cleared after one cycle by opening the transmission line connecting buses 16 and 17. The machines are modeled using the subtransient model with a DC1A exciter, PSS1A stabilizer, and IEEE-G3 governor. Fig. 4 shows the estimated generator 5 internal rotor speed by DSE, numerical derivative with low pass filter and numerical derivative with washout filter [56] of the derived generator angle from the terminal voltage and current measurements. As expected, the numerical derivative of the approximated rotor angle suffers from numerical errors at the moment the event happens; numerical derivative with washout filter is able to address that but at the cost of modifying the time-domain response. By contrast, the DSE can accurately track the rotor speed. On the other hand, ROCoF has been utilized as an important parameter for system protection, especially for the inverter-based generations. By treating it as a dynamic state instead of an algebraic variable, an accurate estimation of it can be achieved [20].

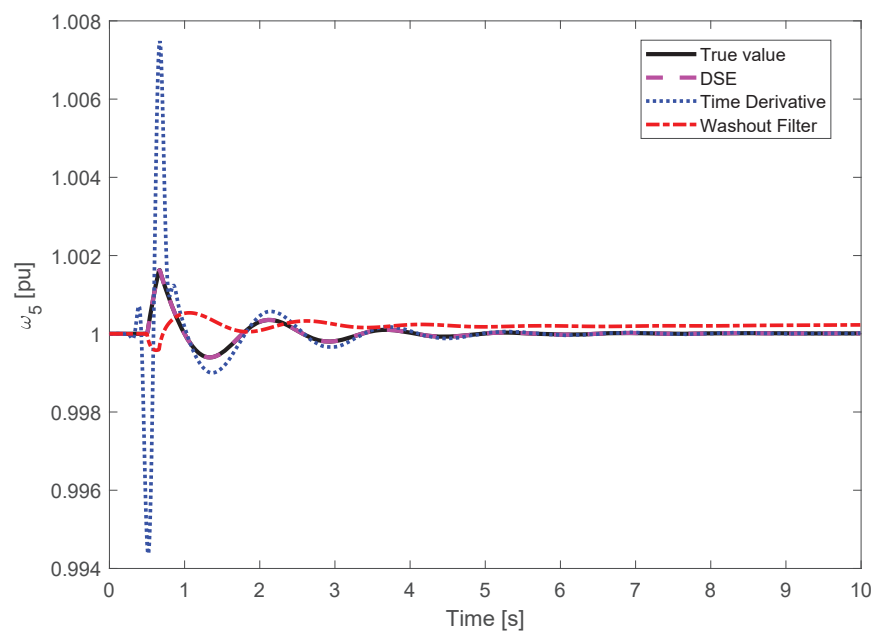

Fig. 4. Comparisons of different methods for internal generator frequency estimation.

The measurements are always subject to noise and even large errors caused by communications, instrument transformer saturation, cyber-attacks, etc. It is important that DSE naturally filters out the measurement noise and provides more accurate data for other applications. Since the field measurements typically follow non-Gaussian distributions [57], robust DSE developed based on the robust statistics theory is needed [58]. While for randomly occurred bad measurements, both normalized residual statistical tests and robust detection are developed. However, it is shown that the threshold for the traditional normalized residual statistical test is not analytical and depends on different systems characteristics [59]. Also, it does not provide reliable outputs in the presence of unknown measurement noise statistics. This is not the case for the robust estimation that automatically detects and suppresses bad data. For the cyber-attack scenario, robust DSE with high breakdown points or novel machine learning-aided DSE might offer a solution [60]. This is still an open area that requires further investigation.

Another important visibility is on the excitation system that could significantly affect the system stability. Although PMU measurements might be used to calculate the excitation voltage in certain cases [61], this cannot be generalized to the modern brushless excitation systems. Furthermore, under stressed conditions, the excitation voltage is restricted by timer-based over-excitation limiters, which may dramatically affect the system stability margin [62]. Traditional DSA model typically does not capture if the state variables corresponding to exciter output are saturated or not. When saturation occurs, it is no longer a state variable and thus should be kept as an unknown input estimation. To achieve this goal, the excitation voltage is taken as the unknown variable for a twostage estimation, where the estimation of input is done first and then substituted into the original model for DSE. Both EKF [63], [64] and UKF [65] considering unknown excitation voltage are used for that and the UKF has a better capability of handling the model nonlinearities and saturation effect. 
These two approaches require the local generator frequency to ensure the observability of unknown inputs. However, the local generator frequency is typically not measured directly by the PMUs. To deal with that, a correlation aided robust DSE for unknown input and state estimation is proposed in [66]. Compared with the previous works, no generator frequency measurement is required, and it has better robustness in dealing with bad data. It is worth noting that the unknown (i.e., not measured) mechanical torque of the turbine-governor system is also estimated using the approach in [66].

Besides the unknown control inputs, there are also anomalies, such as controller failures or malfunctions that can affect the system stability. For example, if the over-excitation occurs, a voltage security issue may arise. Furthermore, if the failure of the excitation system happens but without being detected, the accuracy of the differential-algebraic equations will be negatively impacted, leading to incorrect conclusions about system stability. It is very important to detect these failures timely to avoid the risk of exciter and voltage regulator damages. By relying on the estimated dynamic states and checking the consistency between the control model outputs and expected outputs, the over-excitation and abnormal mechanical power changes can be detected [67]. The multiple models based DSE technique is also developed to detect excitation failures in [31]. Those techniques may be further extended to deal with the anomalies of other controllers, such as governor, and power system stabilizer.

There are other DSE-based monitoring applications that use DAEs but with much larger time constants as compared to electromechanical dynamics. This is the case, for instance, of the increasingly important real-time thermal rating of lines and cables, which is drastically changing the customary way in which static security assessment is performed. Indeed, the existing assets are reaching their conservative ampacity limits, typically defined on a seasonal basis, without due consideration to meteorological conditions (temperature, wind speed, etc.). The simultaneous, real-time estimation of the external parameters arising in the thermal models of lines and cables can help us improve the operation of underground cables [68], [69].

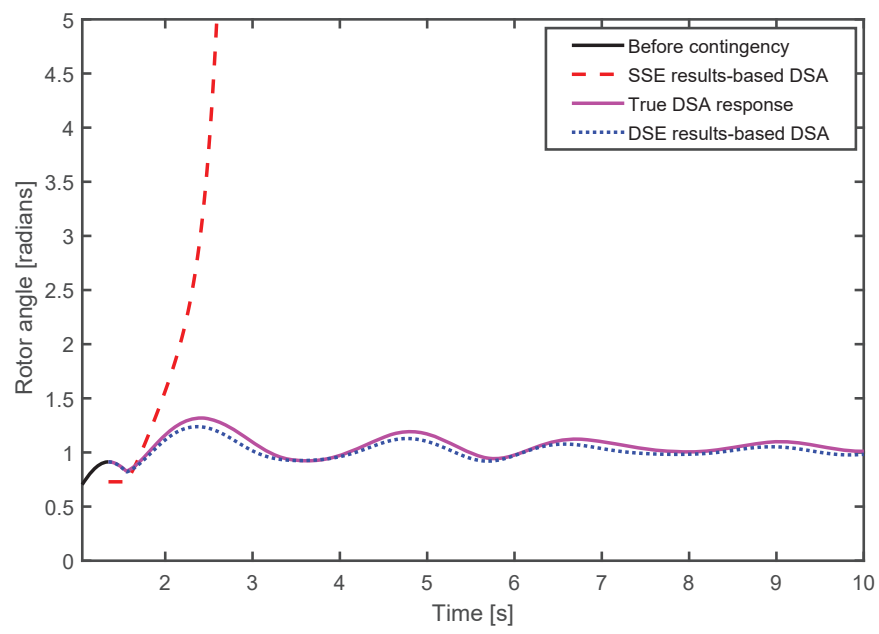

Fig. 5. Influence of initial conditions on DSA results.

\section{Operation}

SSE plays an important role in today's power system operations, especially the essential data base for contingency analysis, static voltage stability and optimal power flow. Compared with SSE, DSE outputs would also offer new benefits to today's EMS. Two main categories are elaborated here, namely DSA and dynamic stability assessment.

Model-based DSA relies on the initialization of dynamic models. The inaccurate initialization of variables might lead to different conclusions about whether the system is stable or not. In the legacy power systems, the operating point evolves slowly in time and the SSE results can be used to calculate the system's initial conditions that are required by DSA tools [16]. In other words, if the system is in equilibrium at $60 \mathrm{~Hz}$ (or $50 \mathrm{~Hz}$ ), SSE is adequate for initializing the differential equations, which is how DSA is done in today's EMS. When the system is not at equilibrium or not at $60 \mathrm{~Hz}$ (or $50 \mathrm{~Hz}$ ), SSE can't be used to initialize the differential equations. Indeed, in future power systems with high penetration of DERs, the variables will change more often and more rapidly, rendering SSE too slow to provide DSA tools with an accurate picture of the system states. But it is during this time period that DSA would be even more important for power system operation. One example is the 2003 Northeast blackout, where the system frequency deviated from $60 \mathrm{~Hz}$ for hours and DSA would help to guide operator's restorative actions. Model initialization for DSA is therefore the first important role of DSE. This is demonstrated through a simple numerical simulation carried out on the IEEE 39-bus system. The system is assumed to be operated under the scenario, where the loads and DERs have large stochastic behaviors, yielding some oscillations of the synchronous generators. The operator would like to assess if the system is able to withstand a contingency. To this end, at $t=1.3 \mathrm{~s}$, a three-phase short-circuit is applied at Bus 28 and cleared after $30 \mathrm{~ms}$ by opening the transmission line connecting Buses 28 and 29. Two cases are considered: 1) the model is initialized by using the estimated bus voltage magnitudes and angles obtained from SSE at $t=1.3 \mathrm{~s} ; 2$ ) the model is initialized by using the dynamic states obtained from DSE at $t=1.3 \mathrm{~s}$. DSA is then performed in both cases. The rotor angle of Generator 9 with respect to that of Generator 10 is displayed in Fig. 5. It can be observed that the SSE-based DSA indicates that the system loses stability while the actual system remains stable. By contrast, the DSE-based DSA reflects true system behavior. The key insight is that in the SSE-based initialization, the derivatives of dynamic state variables are set to zeros based on the quasi-steady-state assumption, but the actual system already deviates from a steady state due to the variations of relative large loads and DERs. As a result, the calculated states using SSE-based DSA are not accurate. This is not the case for DSE-based DSA as their results contain the non-zero derivatives and can be directly used for non quasi-steady-state initialization.

Dynamic stability assessment typically relies on a database that is built from offline simulations, wherein the evolution of the system dynamic states following different events is recorded. Then, an online classifier algorithm, e.g. decision 

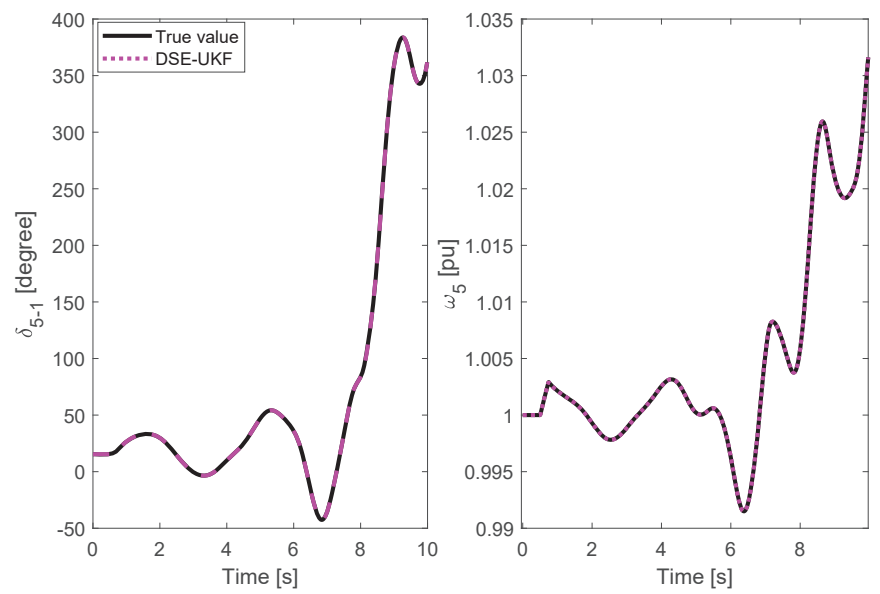

Fig. 6. DSE for unstable system with high non-linearity.

tree or random forest, continuously compares real-time measurements with the database. If an evolving instability is detected, remedial control actions are initiated. This control philosophy is deployed in many power systems around the world. Due to its dependence on offline simulations, it is challenging to consider all possible operating scenarios during offline simulations [70]. This is particularly important with the increasing deployment of power electronics-interfaced DERs. DSE offers an alternative to these databases as it can provide a picture of the system dynamics in almost real-time, and can be used to compute transient stability indices, e.g., rotor angle stability [71]. This alternative control philosophy based on real-time information is yet in its infancy but seems to be promising. Rotor angle stability can also be assessed via the sign of the system's maximal Lyapunov exponent [33], where the generator rotor angles provided by DSE would be of importance to predict system stability. To further demonstrate the capability of DSE in dealing with system instability, the same three-phase fault for the results in Fig. 4 is used but with 30 cycles of clearing time. This leads to system instability as shown in Fig. 6. The robust UKF-based DSE [14] is utilized to estimate and track generator rotor angle and speed. We observe that even on the verge of losing stability, where the system response is highly nonlinear, DSE continues to track the evolution of the system states that can be used for emergency/ in extremis control schemes. On the other hand, DSE could benefit voltage stability assessment. From the short-term voltage stability perspective, DSE allows us to monitor the online states of synchronous generators' excitation system and induction generators/motors, and carry out stability assessment and controls. From the long-term voltage stability perspective, DSE can provide enhanced visibility of system states and snapshots [72]. In particular, the monitoring of the overexcitation limiters, and the detection of the failure of the excitation system and the topology changes would result in better early detection of voltage instability issues. For future power electronics-dominated systems, the frequency issue is of critical concern due to the reduced system inertia. DSE can provide online frequency information to enable a more effective system frequency stability assessment. However, there are very limited researches along these directions and more investigations are needed.

\section{DSE for Power Electronics-Interfaced Renewable Genera- tion Visibility}

Renewable energy sources (RESs) are typically integrated with the grid through power electronics converters. To achieve reliable and cost-effective RESs integration, dynamic visibility is needed. Compared with the conventional synchronous generators, RESs exhibit a higher level of variability and uncertainty. Furthermore, the power converters and the control loops of RESs are very different from the synchronous machines. The power electronics have much smaller time constants than those of the synchronous generators, yielding much faster dynamics. As a result, the measurement system used for the monitoring of RESs should have a faster sampling rate and broader bandwidths for data transmission. It should be noted that besides the traditional voltage stability, rotor angle stability and frequency stability, there is also converter-induced stability [73]. For monitoring and control such fast dynamics of the converters, DSE would be a good choice. For example, the UKF-based DSE is developed to extract the fundamental components of the point of common coupling voltage and load current for grid synchronization of PV systems in [74]. The doubly fed induction generator (DFIG) wind generator's model can be put into the statespace form with their dynamic states estimated by DSE to achieve visibility. The DSE can be implemented via UKF, particle filter, and unscented particle filter [75]-[77]. Since there are errors with the wind speed measurements, DSE for DFIG with unknown wind speeds is addressed in [78]. The joint estimation of dynamic states and parameters of permanent-magnet synchronous motor-based wind generator is investigated in [79]. It should be noted that the relation of estimated states via DSE with system dynamics and stability phenomena is still the subject of ongoing research. Except for the visibility of dynamic states, it is also important to detect any anomalies inside the RESs, such as control failures, erroneous tripping actions, etc. This would provide timely information for operators to take proactive control actions for maintaining system stability. For example, due to the erroneous frequency and ROCOF measurements provided by the phaselocked-loop, several large solar farms have been incorrectly tripped in the 2016 California blue cut fire event [80]. The DSE would allow us to provide more accurate frequency and ROCOF estimates and visibility of abnormal behaviors by checking the model and measurement consistency. This is an open problem that needs more investigations.

\section{CONClusions AND Future Work}

This paper provides a comprehensive summary of the roles of DSE for power system modeling, monitoring and operation. The relationships between SSE and DSE have been identified from the implementation requirements and related EMS functionalities. Several representative examples have been presented to highlight the critical importance of DSE for future power systems, especially for the development of the next-generation EMS. 
Future research on DSE can be generally categorized into four key aspects: data infrastructure for DSE, DSE core functions, DSE applications, and its practical implementation to support the operation and planning of future power systems.

- Data Infrastructure for DSE: DSE, whether being implemented in a centralized or distributed/decentralized manner, needs real-time measurements, and these measurements need to be transferred to proper locations, such as a control center, a generation facility, or a substation. The power industry has been moving forward in the deployment of both sensors and communication networks. Research topics include: what are the data requirements such as data rates, signals, sensor placement? What are the data communication requirements, such as bandwidth, reliability, and redundancy for wide-area system applications? How to achieve more efficient and robust parallel and/or distributed implementations of DSE? And for a hierarchical system, how would the data communication network be structured and where data should be sent to for the DSE application?

- DSE Core Functions: With the active efforts by many researchers for more than a decade, many DSE algorithms have been developed, and the DSE performance has been significantly improved, as summarized in this paper. However, power systems are evolving to be more complex and different, and therefore DSE core functions should continue to improve to meet the operational requirements of future power systems. Research topics include: How to improve computational aspects of DSE to meet real-time requirements for large-scale systems? How to formulate DSE with fast inverter dynamics and potentially deal with mixed slow and fast dynamics? Can machine learning or other data analytical approaches help improve DSE performance in the context of a large amount of data and a large number of system configurations? And how to break DSE into pieces in real-time for islanding situations, especially for a more resilient power system?

- Development of DSE Applications: DSE provides unprecedented detailed dynamic information, compared with SSE. A large number of applications in modeling, monitoring and operation can benefit from DSE as mentioned in this paper. Many more are emerging. There are conventional applications that can be enhanced by DSE-provided dynamic information and new applications that can be enabled by DSE. Some of them include: aggregated model calibration for wind/solar farms, loads and DERs; oscillation source location; look-ahead DSA; visibility and detection of converter-induced instability; anomaly detection of DERs, where the complicated control loop may have fault or failures etc.

- Practicality of DSE Applications: All the research efforts have a common goal which is to make DSE a practical part of the power system functions. Model calibration is an example of DSE applications, which is already in commercial tools, reliability standards, and industry practices. For most DSE applications, there are still significant gaps to address in terms of their practicality.
Research questions include: How to transition from SSE to DSE while SSE and DSE will most likely co-exist for a significant period? How to make DSE compatibility with the control room environment in terms of its information technology infrastructure and human-machine interface? For example, DSE results are updated too fast for operators to take actions and how to present the critical information only to operators would be an important research need. This may be addressed by development of advanced AI tools fed with DSE data streaming for visibility and stability assessment; What training should be developed and provided to prepare the workforce for DSE applications? And what new standards are needed to enable the DSE applications?

\section{ACKNOWLEDGEMENT}

The authors would like to thank all IEEE TF members for their valuable discussions and suggestions during web and face-to-face meetings. We also thank the anonymous reviewers for helping us to improve the quality of the paper.

\section{REFERENCES}

[1] A. Abur, Dynamic-State Estimation. Wiley, 2016, pp. 1-14.

[2] B. Kroposki et al., "Achieving a 100\% renewable grid: Operating electric power systems with extremely high levels of variable renewable energy," IEEE Power and Energy Magazine, vol. 15, no. 2, pp. 61-73, Mar.-Apr. 2017.

[3] P. W. Sauer, M. A. Pai, and J. H. Chow, Power System Dynamics and Stability: With Synchrophasor Measurement and Power System Toolbox 2e. John Wiley \& Sons, Ltd, 2017.

[4] J. Zhao et al., "Power system dynamic state estimation: Motivations, definitions, methodologies, and future work," IEEE Trans. Power Syst., vol. 34, no. 4, pp. 3188-3198, Jul. 2019.

[5] W. Miller and J. Lewis, "Dynamic state estimation in power systems," IEEE Trans. Automatic Control, vol. 16, no. 6, pp. 841-846, Dec. 1971.

[6] J. De La Ree, V. Centeno, J. S. Thorp, and A. G. Phadke, "Synchronized phasor measurement applications in power systems," IEEE Trans. Smart Grid, vol. 1, no. 1, pp. 20-27, Jun. 2010.

[7] E. Y. Song, K. B. Lee, G. J. FitzPatrick, and Y. Zhang, "Interoperability test for IEC 61850-9-2 standard-based merging units," in IEEE Power and Energy Society Innovative Smart Grid Technologies Conference, 2017, pp. 1-6.

[8] Z. Huang, K. Schneider, and J. Nieplocha, "Feasibility studies of applying Kalman filter techniques to power system dynamic state estimation," in International Power Engineering Conference, 2007, pp. 376-382.

[9] E. Ghahremani and I. Kamwa, "Online state estimation of a synchronous generator using unscented Kalman filter from phasor measurements units,' IEEE Trans. Energy Conversion, vol. 26, no. 4, pp. 1099-1108, Dec. 2011.

[10] N. Zhou, D. Meng, Z. Huang, and G. Welch, "Dynamic state estimation of a synchronous machine using PMU data: A comparative study," IEEE Trans. Smart Grid, vol. 6, no. 1, pp. 450-460, Jan. 2015.

[11] M. Netto and L. Mili, "A robust data-driven Koopman Kalman filter for power systems dynamic state estimation," IEEE Trans. Power Syst., vol. 33, no. 6, pp. 7228-7237, Nov. 2018.

[12] A. Rouhani and A. Abur, "Observability analysis for dynamic state estimation of synchronous machines," IEEE Trans. Power Syst., vol. 32, no. 4, pp. 3168-3175, Jul. 2017.

[13] J. Zhao, M. Netto, and L. Mili, "A robust iterated extended Kalman filter for power system dynamic state estimation," IEEE Trans. Power Syst., vol. 32, no. 4, pp. 3205-3216, Jul. 2017.

[14] J. Zhao and L. Mili, "A theoretical framework of robust H-infinity unscented Kalman filter and its application to power system dynamic state estimation," IEEE Trans. Signal Processing, vol. 67, no. 10, pp. 2734-2746, May 2019.

[15] A. Rouhani and A. Abur, "Linear phasor estimator assisted dynamic state estimation," IEEE Trans. Smart Grid, vol. 9, no. 1, pp. 211-219, Jan. 2018. 
[16] L. Wang and K. Morison, "Implementation of online security assessment," IEEE Power and Energy Magazine, vol. 4, no. 5, pp. 46-59, Sept.-Oct. 2006.

[17] Z. Huang, R. Diao, S. Jin, and Y. Chen, "Predictive dynamic security assessment through advanced computing," in IEEE PES General Meeting, 2014, pp. $1-5$.

[18] "IEEE recommended practice for excitation system models for power system stability studies," IEEE Std 421.5-2016 (Revision of IEEE Std 421.5-2005), pp. 1-207, Aug. 2016.

[19] H. Su, R. Mutukutti, and D. Apps, "Impacts of variable quadrature reactance on power system stabilizer performance," in IEEE Power and Energy Society General Meeting, 2013, pp. 1-5.

[20] A. K. Singh and B. C. Pal, "Rate of change of frequency estimation for power systems using interpolated DFT and Kalman filter," IEEE Trans. Power Syst., vol. 34, no. 4, pp. 2509-2517, Jul. 2019.

[21] J. Qi, K. Sun, and W. Kang, "Optimal PMU placement for power system dynamic state estimation by using empirical observability Gramian," IEEE Trans. Power Syst., vol. 30, no. 4, pp. 2041-2054, Jul. 2015.

[22] A. Abur and A. G. Expósito, Power System State Estimation: Theory and Application. CRC Press, 2004.

[23] N. Zhou, S. Wang, J. Zhao, and Z. Huang, "Application of detectability analysis for power system dynamic state estimation," IEEE Trans. Power Syst., vol. 35, no. 4, pp. 3274-3277, Jul. 2020.

[24] J. Zhang, G. Welch, G. Bishop, and Z. Huang, "Optimal PMU placement evaluation for power system dynamic state estimation," in IEEE PES Innovative Smart Grid Tech., 2010, pp. 1-7.

[25] J. B. Zhao, L. Mili, R. C. Pires, "Statistical and numerical robust state estimator for heavily loaded power systems," IEEE Trans. Power Syst., vol. 33, no. 6, 6904-6914, Nov. 2018.

[26] J. Chen, Y. Liao, and B. Gou, "Study of WLS state estimation convergence characteristics under topology errors," in Proc. IEEE Southeastcon, Jacksonville, FL, USA, 2013, pp. 1-5.

[27] M. Boutayeb, H. Rafaralahy, M. Darouach, "Convergence analysis of the extended Kalman filter used as an observer for nonlinear deterministic discrete-time systems," IEEE Trans. Automatic Control, vol. 42, no. 4, pp. 581-586, Apr. 1997.

[28] A. Daid, E. Busvelle, and M. Aidene, "On the convergence of the unscented Kalman filter," European Journal of Control, 2020.

[29] J. Mandel, L. Cobb, J. Beezley, "On the convergence of the ensemble Kalman filter," Applications of Mathematics, vol. 56, no. 6, pp. 533-541, 2011.

[30] M. Göl and A. Abur, "LAV based robust state estimation for systems measured by PMUs," IEEE Trans. Smart Grid, vol. 5, no. 4, pp. 18081814, Jul. 2014

[31] A. Rouhani and A. Abur, "A robust dynamic state estimator against exciter failures," in North American Power Symposium, 2016, pp. 1-6.

[32] G. Anagnostou, F. Boem, S. Kuenzel, B. C. Pal, and T. Parisini, "Observer-based anomaly detection of synchronous generators for power systems monitoring," IEEE Trans. Power Syst., vol. 33, no. 4, pp. 42284237, Jul. 2018

[33] S. Wei et al., "Model-free MLE estimation for online rotor angle stability assessment with PMU data," IEEE Trans. Power Syst., vol. 33, no. 3, pp. 2463-2476, May 2018.

[34] A. P. S. Meliopoulos et al., "Dynamic state estimation-based protection: Status and promise," IEEE Trans. Power Delivery, vol. 32, no. 1, pp. 320-330, Feb. 2017.

[35] J. Zhao and L. Mili, "A robust generalized-maximum likelihood unscented Kalman filter for power system dynamic state estimation," IEEE Journal of Selected Topics in Signal Processing, vol. 12, no. 4, pp. 578592, Aug. 2018

[36] "Reliability guideline: Power plant dynamic model verification using PMUs," North American Electric Reliability Corporation (NERC), Tech. Rep., Sept. 2016.

[37] Z. Huang, P. Du, D. Kosterev, and S. Yang, "Generator dynamic model validation and parameter calibration using phasor measurements at the point of connection," IEEE Trans. Power Syst., vol. 28, no. 2, pp. 19391949, May 2013.

[38] R. Huang et al., "Calibrating parameters of power system stability models using advanced ensemble Kalman filter," IEEE Trans. Power Syst., vol. 33, no. 3, pp. 2895-2905, May 2018.

[39] S. Akhlaghi, S. Raheem, and N. Zhou, "Model validation lessons learned through implementing NERC MOD-033-1," in IEEE Power and Energy Society General Meeting, 2020, pp. 1-5.

[40] G. Valverde, E. Kyriakides, G. T. Heydt, and V. Terzija, "Nonlinear estimation of synchronous machine parameters using operating data," IEEE Trans. Energy Conversion, vol. 26, no. 3, pp. 831-839, Sept. 2011.
[41] M. A. Gonzalez-Cagigal, J. A. Rosendo-Macias, A. Gomez-Exposito, "Parameter estimation of fully regulated synchronous generators using unscented Kalman filters", Electr. Power Syst. Res., vol. 168, pp. 1137-1144, Sep., 2019.

[42] A. Rouhani and A. Abur, "Constrained iterated unscented Kalman filter for dynamic state and parameter estimation," IEEE Trans. Power Syst., vol. 33, no. 3, pp. 2404-2414, May 2018.

[43] M. A. M. Ariff, B. C. Pal, and A. K. Singh, "Estimating dynamic model parameters for adaptive protection and control in power system," IEEE Trans. Power Syst., vol. 30, no. 2, pp. 829-839, Mar. 2015.

[44] P. Bhui, N. Senroy, A. K. Singh, and B. C. Pal, "Estimation of inherent governor dead-band and regulation using unscented Kalman filter," IEEE Trans. Power Syst., vol. 33, no. 4, pp. 3546-3558, Jul. 2018.

[45] A. Rouhani and A. Abur, "Real-time dynamic parameter estimation for an exponential dynamic load model," IEEE Trans. Smart Grid, vol. 7, no. 3, pp. 1530-1536, May 2016.

[46] Y. Zhang, E. Muljadi, D. Kosterev, and M. Singh, "Wind power plant model validation using synchrophasor measurements at the point of interconnection," IEEE Trans. Sustainable Energy, vol. 6, no. 3, pp. 984-992, Jul. 2015.

[47] R. Fan, Z. Huang, S. Wang, R. Diao, and D. Meng, "Dynamic state estimation and parameter calibration of a DFIG using the ensemble Kalman filter," in IEEE Power and Energy Society General Meeting, 2015, pp. $1-5$

[48] "Parameter estimation of fully regulated synchronous generators using unscented Kalman filters."

[49] S.-K. Joo, C.-C. Liu, L. E. Jones, and J.-W. Choe, "Coherency and aggregation techniques incorporating rotor and voltage dynamics," IEEE Trans. Power Syst., vol. 19, no. 2, pp. 1068-1075, May 2004.

[50] M. Aghamohammadi and S. Tabandeh, "A new approach for online coherency identification in power systems based on correlation characteristics of generators rotor oscillations," International Journal of Electrical Power \& Energy Systems, vol. 83, pp. 470 - 484, Dec. 2016.

[51] A. Chakrabortty, J. H. Chow, and A. Salazar, "A measurement-based framework for dynamic equivalencing of large power systems using wide-area phasor measurements," IEEE Trans. Smart Grid, vol. 2, no. 1, pp. 68-81, Mar. 2011.

[52] "Identification of electromechanical modes in power systems," IEEE Power and Energy Society, Power System Dynamic Performance Committee (PSDP), Tech. Rep., Jun. 2012.

[53] S. Maslennikov et al., "A test cases library for methods locating the sources of sustained oscillations," in IEEE Power and Energy Society General Meeting, 2016, pp. 1-5.

[54] J. Zhao, L. Mili, and F. Milano, "Robust frequency divider for power system online monitoring and control," IEEE Trans. Power Syst., vol. 33, no. 4, pp. 4414-4423, Jul. 2018 .

[55] J. Zhao, Y. Tang, and V. Terzija, "Robust online estimation of power system center of inertia frequency," IEEE Trans. Power Syst., vol. 34 no. 1, pp. 821-825, Jan. 2019

[56] F. Milano and Á. Ortega, "Frequency divider," IEEE Trans. Power Syst., vol. 32, no. 2, pp. 1493-1501, Mar. 2017

[57] S. Wang, J. Zhao, Z. Huang, and R. Diao, "Assessing Gaussian assumption of PMU measurement error using field data," IEEE Trans. Power Delivery, vol. 33, no. 6, pp. 3233-3236, Dec. 2018.

[58] J. Zhao, L. Mili, and A. Gómez-Expósito, "Constrained robust unscented Kalman filter for generalized dynamic state estimation," IEEE Trans. Power Syst., vol. 34, no. 5, pp. 3637-3646, Sept. 2019.

[59] J. Zhao, L. Mili, "Robust unscented Kalman filter for power system dynamic state estimation with unknown noise statistics," IEEE Trans. Smart Grid, vol. 10, no. 2, pp. 1215-1224, 2019.

[60] Y. Chakhchoukh, H. Lei, and B. K. Johnson, "Diagnosis of outliers and cyber attacks in dynamic PMU-based power state estimation," IEEE Trans. Power Syst., vol. 35, no. 2, pp. 1188-1197, Mar. 2020.

[61] Q. Yang, T. Bi, and J. Wu, "WAMS implementation in China and the challenges for bulk power system protection," in IEEE Power Engineering Society General Meeting, 2007, pp. 1-6.

[62] G. Anagnostou and B. C. Pal, "Impact of overexcitation limiters on the power system stability margin under stressed conditions," IEEE Trans. Power Syst., vol. 31, no. 3, pp. 2327-2337, May 2016.

[63] E. Ghahremani and I. Kamwa, "Dynamic state estimation in power system by applying the extended Kalman filter with unknown inputs to phasor measurements," IEEE Trans. Power Syst., vol. 26, no. 4, pp. 2556-2566, Nov. 2011.

[64] E. Ghahremani and I. Kamwa, "Local and wide-area PMU-based decentralized dynamic state estimation in multi-machine power systems," IEEE Trans. Power Syst., vol. 31, no. 1, pp. 547-562, Jan. 2016. 
[65] G. Anagnostou and B. C. Pal, "Derivative-free Kalman filtering based approaches to dynamic state estimation for power systems with unknown inputs," IEEE Trans. Power Syst., vol. 33, no. 1, pp. 116-130, Jan. 2018.

[66] J. Zhao et al., "Correlation-aided robust decentralized dynamic state estimation of power systems with unknown control inputs," IEEE Trans. Power Syst., vol. 35, no. 3, pp. 2443-2451, May 2020.

[67] P. Marchi, P. Gill Estevez, F. J. Messina, and C. G. Galarza, "Loss of excitation detection in synchronous generators based on dynamic state estimation," IEEE Trans. Energy Conversion, pp. 1-1, 2020.

[68] M. Diaz-Aguiló and F. de León, "Adaptive soil model for real-time thermal rating of underground power cables," IET Science, Measurement \& Technology, vol. 9, no. 6, pp. 654-660, 2015.

[69] A. Gómez-Expósito et al., "City-friendly smart network technologies and infrastructures: The Spanish experience," Proceedings of the IEEE, vol. 106, no. 4, pp. 626-660, Apr. 2018.

[70] I. L. Ortega Rivera, V. Vittal, G. T. Heydt, C. R. Fuerte-Esquivel, and C. Angeles-Camacho, "A dynamic state estimator based control for power system damping," IEEE Trans. Power Syst., vol. 33, no. 6, pp. 6839-6848, Nov. 2018.

[71] A. Paul, I. Kamwa, and G. Joos, "PMU signals responses-based RAS for instability mitigation through on-the fly identification and shedding of the run-away generators," IEEE Trans. Power Syst., vol. 35, no. 3, pp. 1707-1717, May 2020.

[72] M. Glavic and T. Van Cutsem, "Wide-area detection of voltage instability from synchronized phasor measurements. Part I: Principle," IEEE Trans. Power Syst., vol. 24, no. 3, pp. 1408-1416, Aug. 2009.

[73] "Stability definitions and characterization of dynamic behavior in systems with high penetration of power electronic interfaced technologies," IEEE Power and Energy Society, Power System Dynamic Performance Committee (PSDP), Tech. Rep., May 2020.

[74] S. Swain and B. Subudhi, "Grid synchronization of a PV system with power quality disturbances using unscented Kalman filtering," IEEE Trans. Sustainable Energy, vol. 10, no. 3, pp. 1240-1247, Jul. 2019.

[75] S. Yu, K. Emami, T. Fernando, H. H. C. Iu, and K. P. Wong, "State estimation of doubly fed induction generator wind turbine in complex power systems," IEEE Trans. Power Syst., vol. 31, no. 6, pp. 4935-4944, Nov. 2016.

[76] S. Yu, T. Fernando, H. H. Iu, and K. Emami, "Realization of stateestimation-based DFIG wind turbine control design in hybrid power systems using stochastic filtering approaches," IEEE Trans. Industrial Informatics, vol. 12, no. 3, pp. 1084-1092, Jun. 2016.

[77] S. S. Yu et al., "An unscented particle filtering approach to decentralized dynamic state estimation for DFIG wind turbines in multi-area power systems," IEEE Trans. Power Syst., vol. 35, no. 4, pp. 2670-2682, Jul. 2020 .

[78] G. Anagnostou, L. P. Kunjumuhammed, and B. C. Pal, "Dynamic state estimation for wind turbine models with unknown wind velocity," IEEE Trans. Power Syst., vol. 34, no. 5, pp. 3879-3890, Sept. 2019.

[79] M. A. González-Cagigal, J. A. Rosendo-Macías, and A. GómezExpásito, "Parameter estimation of wind turbines with PMSM using cubature Kalman filters," IEEE Trans. Power Syst., vol. 35, no. 3, pp 1796-1804, May 2020.

[80] "1,200 MW fault induced solar photovoltaic resource interruption disturbance report: Southern California 8/16/2016 event," North American Electric Reliability Corporation (NERC), Tech. Rep., Jun. 2017. 